\title{
Magnetodielectric Properties of Transformer Oil Based Magnetic Fluids
}

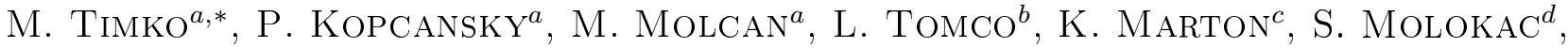

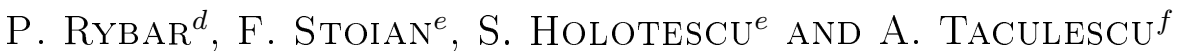 \\ ${ }^{a}$ Institute of Experimental Physics, Slovak Academy of Sciences, 04001 Kosice, Slovakia \\ ${ }^{b}$ Faculty of Aeronautic, Technical University in Kosice, 04121 Kosice, Slovakia \\ ${ }^{c}$ Faculty of Electrical Engineering and Informatics, Technical University in Kosice, 04200 Kosice, Slovakia \\ ${ }^{d}$ Faculty of Mining, Ecology, Process Control and Geotechnology, TU Kosice, Letna 9, 04200 Kosice, Slovakia \\ ${ }^{e}$ Politehnica University of Timisoara, Timisoara 300222, Romania \\ ${ }^{f}$ Laboratory of Magnetic Fluids, Romanian Academy — Timisoara Division, Timisoara, Romania
}

\begin{abstract}
This contribution is devoted to study of dielectric and magnetodielectric properties in transformer oil based magnetic fluid. The dielectric permittivity and their anisotropy at various mutual orientation electric $(50 \mathrm{~Hz})$ and magnetic field were shown at the different volume concentrations of nanoparticles at room temperature. The linear increase of dielectric constant with volume concentration was confirmed which is in good agreement with the theoretical Maxwell prediction. The dielectric anisotropy factor $g(B, \omega)$ is very close to $g=1$. The values of permittivity increased with the increase of volume fraction of magnetite nanoparticles. The highest value of anisotropy characterized by deviation of permittivity $\Delta \varepsilon=\varepsilon_{\|}-\varepsilon_{\perp}$ was found for the highest volume concentration which could mean that no aggregation of magnetite nanoparticles had appeared.
\end{abstract}

PACS: $47.65 . \mathrm{Cb}, 77.22 . \mathrm{Gm}, 77.22 . \mathrm{Ch}$

\section{Introduction}

Magnetic fluids are mixtures of organic solvents and ferro or ferrimagnetic particles in essence. But the important factor is that the particles are uniformly dispersed throughout the liquid volume. Their specific properties are important for use in various fields, from the technical sciences to biosciences. There is interest in applied magnetic fluids based on transformer oil in order to improve the operating parameters of power transformers. From magnetic fluids are expected to be better thermal and dielectric benefits to transformers. This follows from improved cooling by enhancing fluid circulation within transformer windings.

Investigation of dielectric and magnetodielectric properties of magnetic fluids is connected intimately with magnetodielectric effect that is indicated by magnetodielectric anisotropy. The dielectric behavior of magnetic fluids changes with the application of an external magnetic field and with the relative orientation of the electric and magnetic fields. This effect is known as magneto-dielectric anisotropy effect [1].

Magneto-dielectric effects in magnetic fluids have been investigated by many workers [2-6]. The experimental in-

* corresponding author; e-mail: timko@saske.sk vestigations were based on impedance measurement techniques where the magnetic fluid is placed in a capacitor. Measurements of the impedance parameters such as the modulus and phase are carried out using a bridge or an RLC meter. The impedance measurement techniques suffer some serious disadvantages such as electrode effects, parasitic impedances, skin depth and accuracy related problems, but the works [1, 7] have determined the magneto-dielectric effect from magneto-optical measurements where the disadvantages suffered by conventional impedance measurement techniques are avoided.

When the magnetic fluid is exposed to presence of magnetic field, the processes in macroscopic surroundings of electrode system and microscopic surroundings between magnetic particles themselves created needle-shape aggregations (clusters) of magnetite particles.

One of the solution ways of these processes is equation that expresses effect of force action of magnetic and electric fields in surroundings. The force causing mobility of particle clusters in electric field is dependent on their weight that is dependent on volume concentration of magnetite particles and local density of magnetic fluids. The Stokes force that expresses effect of dynamic viscosity of magnetic fluids, is not negligible at application of alternating electric field with frequency $50 \mathrm{~Hz}$. Velocity of particles $v$ and orientation of its components $\left(v_{\|}\right.$, respectively $\left.v_{\perp}\right)$ relative to vector $\boldsymbol{E}$, pertinently to 
vector $\boldsymbol{B}$ have important role from point of view of magnetic field action with induction $B$. It can be suggested the solution of the following cases:

a) If $\boldsymbol{E}$ is variable and $B=0 \mathrm{~T}$, then force in electric field has as a consequence mechanical stress (compressively, tensile) in magnetic fluids. If we accept hypothesis about local differential changes of permittivity and density caused by action of strong electric field, then there exists a volume force due to the heterogeneous field and anisotropy of insulator medium. Investigation of origin and existence of space charge between electrodes shows [5] that action of electric field causes polarization of magnetic fluid components in nanometric range. As a consequence of this process the gradient force affecting the polarized particles exist.

b) Simultaneous influence of electric and magnetic fluid on magnetodielectric properties of magnetic fluids is devoted to analyze of cases that show what application of electric and magnetic field will be dominated for determination of magnetic fluids permittivity and their loss parameter at respect of the observed medium anisotropy. There have been observed the cases when vectors $\boldsymbol{E}$ and $\boldsymbol{B}$ were parallel and perpendicular $(\boldsymbol{E} \| \boldsymbol{B}, \boldsymbol{E} \perp \boldsymbol{B})$. That is why there is a division of particles velocity $v$ and intensity of electric field $\boldsymbol{E}$ into components parallel and perpendicular to magnetic induction $B\left(v=v_{\|}+v_{\perp}\right.$ and $\left.E=E_{\|}+E_{\perp}\right)$. It can be showed that charged particles motion in the case $\boldsymbol{E} \perp \boldsymbol{B}$ is characterized by two types of motion: drift with constant velocity $v_{E}$ and circle motion with velocity $v_{\perp}$ that is caused only by magnetic field. If $v_{E}$ exists, then particles move in perpendicular direction to vectors $\boldsymbol{E}$ and $\boldsymbol{B}$ in combined electric and magnetic field that causes particles motion on a spiral.

In general, magnetodielectric anisotropy factor is defined as:

$$
g(B, \omega)=-\frac{\varepsilon_{\| \mid}(B, \omega)-\varepsilon(0, \omega)}{\varepsilon_{\perp}(B, \omega)-\varepsilon(0, \omega)},
$$

where $\varepsilon_{\|}(B)$ and $\varepsilon_{\perp}(B)$ are permittivity for $E \| B$ and $E \perp B$ and $\varepsilon(0)$ for $B=0$. The value of $g$ was either 2 or 1 [8-10]. Experimentally, Cotae [5] and Espurz et al. [1] have obtained the value of 1 for $g(H, \omega)$ while Mailfert and Nahounou [2] have obtained a value of 2 . Experimental results of Espurz et al. [1] show that the value of $g(H, \omega)$ is 1 at low fields and 1.23 at high fields.

The present work is devoted to studying the dielectric and magneto-dielectric properties of transformer oil based magnetic fluids depending on concentration of magnetite nanoparticles and mutual orientation of magnetic and electrical fields.

\section{Materials and methods}

The used magnetic fluid was prepared, based on transformer oil UTR 40 and magnetite $\left(\mathrm{Fe}_{3} \mathrm{O}_{4}\right)$ nanoparticles, with oleic acid as surfactant. The synthesis of MNF with organic liquid carrier, based on an established procedure [11], followed these main steps: synthesis of surface coated magnetite nanoparticles: co- -precipitation (at $t \approx 80^{\circ} \mathrm{C}$ ) of magnetite from aqueous solutions of $\mathrm{Fe}^{3+}$ and $\mathrm{Fe}^{2+}$ ions in the presence of concentrated $\mathrm{NH}_{4} \mathrm{OH}$ solution $(25 \%)$, sterical stabilization (chemisorption of oleic acid; $80-82{ }^{\circ} \mathrm{C}$ ), magnetic decantation and repeated washing, extraction of monolayer covered magnetite nanoparticles (acetone added; extraction), to obtain stabilized magnetite nanoparticles. Then, the magnetic nanoparticles were dispersed in the non-polar liquid carrier at $t \approx 120-130^{\circ} \mathrm{C}$, followed by magnetic decantation/filtration, flocculation and re-dispersion of magnetic nanoparticles to obtain the non- polar magnetic nanofluid.

The magnetization curves of the samples were measured by vibrating sample magnetometry using a VSM magnetometer (Model 880, DMS/ADE Technologies, USA) at room temperature $\left(\approx 25^{\circ} \mathrm{C}\right)$ in magnetic field up to $800 \mathrm{kA} \mathrm{m}^{-1}$. The measurement of relative permittivity was carried out with help of Shering bridge Tettex 2818 at frequency $50 \mathrm{~Hz}$. That was controlled by microprocessor. A capacitor was composed from parallel plate $\mathrm{Cu}$ electrodes placed in a container. The electrodes were $2 \mathrm{~cm}$ in diameter and the distance between them was $0.8 \mathrm{~mm}$. Capacity and loss factor of the capacitor were measured as a function of the applied electric field intensity in the range of $0.2-1.2 \mathrm{MV} \mathrm{m}^{-1}$. The experimental error of capacity measurements was $0.05 \%$ and of loss factor was $1 \%$.

Permittivity of magnetic liquids was determined from capacity measurements as $\varepsilon_{r}=C / C_{0}$, where $C$ is the capacity of the capacitor with a magnetic liquid as a dielectric and $C_{0}$ is the capacity of the same capacitor filled by air.

Two permanent $\mathrm{NdFeB}$ magnets were used as a source of homogeneous magnetic field up to $40 \mathrm{mT}$. Four magnetic fluids based on transformer oil with various concentrations of magnetite nanoparticles up to $3.03 \%$ magnetic volume fraction and pure transformer oil have been used in measurements. Gap between circular electrodes was $0.5 \mathrm{~mm}$, temperature $20^{\circ} \mathrm{C}$ and voltage in range from $100 \mathrm{~V}$ to $600 \mathrm{~V}$, i.e. intensity of electric field was from $0.2 \mathrm{MV} \mathrm{m}^{-1}$ to $1.2 \mathrm{MV} \mathrm{m}^{-1}$.

\section{Results and discussion}

There were investigated four samples of magnetic nanofluid, with carrier liquid of transformer oil TR40 and magnetite nanoparticles as magnetic dispersed phase, prepared according to the method presented in [11]. The four samples of different volume fractions MF1 (1.62\%), MF2 (2.15\%), MF3 (2.69\%), and MF4 (3.03\%) were obtained by dilution of the most concentrated one with the carrier liquid. The saturation magnetization measured by VSM was $2.78 \mathrm{kA} / \mathrm{m}, 4.93 \mathrm{kA} / \mathrm{m}, 7.71 \mathrm{kA} / \mathrm{m}$, and $9.07 \mathrm{kA} / \mathrm{m}$ for $1.62 \%, 2.15 \%, 2.69 \%$, and $3.03 \%$, respectively. The additional basic physical properties are given in $[12]$.

The measurements of permittivity showed that value of the permittivity increases with volume concentration 


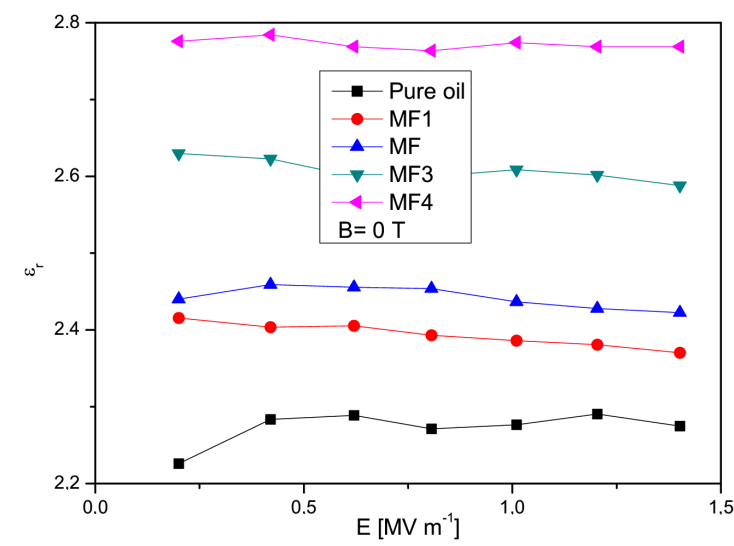

Fig. 1. Dependence of permittivity $\varepsilon_{r}$ of the magnetic liquid on electric field intensity $E$ without magnetic field.

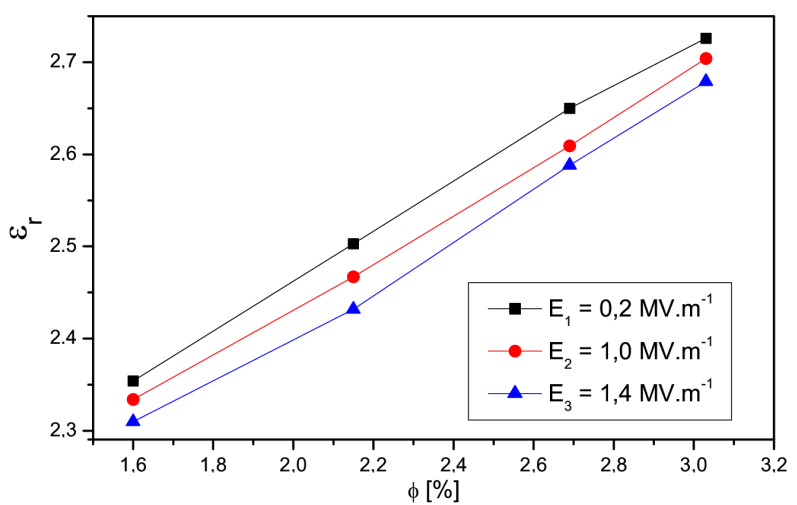

Fig. 2. Dependence of permittivity $\varepsilon_{r}$ of the magnetic liquid on volume concentration at various electric field as a parameter.

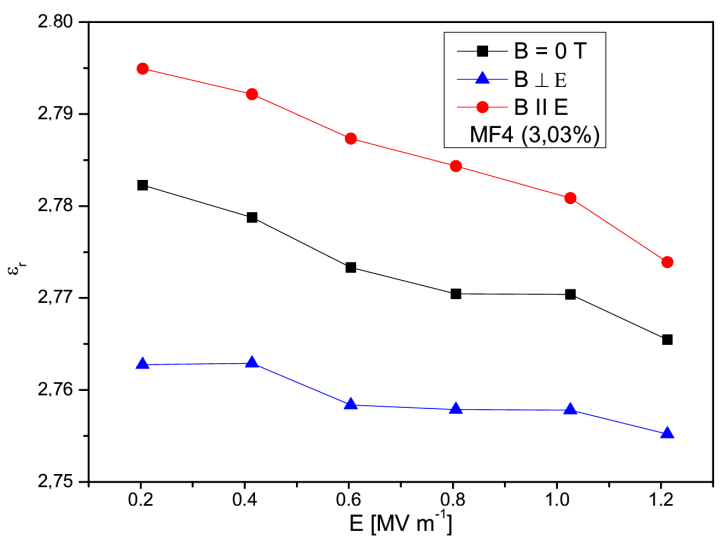

Fig. 3. Dependence of permittivity $\varepsilon_{r}$ of the magnetic liquid on electric field intensity $E$ for sample with volume concentration $3.03 \%$ in parallel and perpendicular orientation $\boldsymbol{E}$ vs. $\boldsymbol{B}$. from 2.23 for $1.62 \%$ up to 2.7 for $3.03 \%$ of magnetic nanoparticles (Fig. 1). Simultaneously the permittivity decreased with increase of the intensity of electric field for all used concentrations of magnetite nanoparticles. The linear dependence of permittivity on volume concentration of nanoparticles was observed, too (Fig. 2), as predicted by the theoretical Maxwell relation [13].

The ferrofluid permittivity recording versus intensity of electric field obtained with an applied external magnetic field is shown in Fig. 3 for magnetic fluid with the volume concentration $3.03 \%$. When continuous magnetic field of $40 \mathrm{mT}$ is applied, the permittivity of the magnetic fluid is increased for parallel and decreased for perpendicular orientation. The induced anisotropy in relative permittivity of a magnetic fluid subjected to an external magnetic field can be seen. The magnetodielectric anisotropy factor $g(B, \omega)$ calculated according to formula (1) is very close to value $g=1$ for all volume concentrations which is in agreement with formerly published results [1].

The variation of the dielectric permittivity with applied magnetic field can be resulting from an alignment of non-interacting nanoparticles as a consequence of the coupling between easy axes and magnetic moment of particles. The similar dependence of dielectric anisotropy was observed for other concentrations, too. The anisotropy characterized by deviation of permittivity $\Delta \varepsilon=\varepsilon_{\|}-\varepsilon_{\perp}$ showed that the highest value was obtained for the highest volume concentration. This fact could mean that no aggregation of magnetite nanoparticle had appeared.

For higher concentrations of magnetite the increase of permittivity with electric field was observed [14]. This increase was explained by existence of cluster formation as magnetite particles coated with oleic acid as a surfactant are electrically charged by adsorbed ions and counter-ions from the surrounding atmosphere may be attracted to them [15]. Counter-ions are ions oppositely charged to ions adsorbed by particles. Electric dipole moments are induced in electric field. Increasing electric field increases electric dipole-dipole interaction between particles and supports their agglomeration.

It was established [15] that in magnetic liquids with nearly spherically shaped particles, the critical frequency exists above which the magnetodielectric effect appears. The critical frequency of transformer oil based magnetic liquid with spherically shaped magnetite particles coated with oleic acid (specific density of sample is $1470 \mathrm{~kg} \mathrm{~m}^{-3}$ and magnetization at $H=8 \times 10^{4} \mathrm{~A} \mathrm{~m}^{-1}$ is $M=$ $25.2 \times 10^{3} \mathrm{~A} \mathrm{~m}^{-1}$ ) reaches a value of $55 \mathrm{~Hz}$ and the critical frequency of kerosene based magnetic liquid is lower at lower specific density [14] therefore we suppose that the critical frequency of our magnetic liquid (specific density is $998 \mathrm{~kg} \mathrm{~m}^{-3}$, magnetization at $H=8 \times 10^{4} \mathrm{~A} \mathrm{~m}^{-1}$ is $M=9.071 \times 10^{3} \mathrm{~A} \mathrm{~m}^{-1}$ is lower than the used measurements frequency of $50 \mathrm{~Hz}$. The existence of magnetodielectric effect was confirmed in our experiments. 
On the other hand, the permittivity measurements in electric and magnetic field confirmed good structural stability of magnetic fluid without aggregation which can predetermine the possibility of its application in power transformer as cooling and isolative medium.

\section{Conclusion}

The magnetodielectric effect in magnetic liquids was confirmed in high electric fields $0.2-1.2 \mathrm{MV} \mathrm{m}^{-1}$ at magnetic field of value $40 \mathrm{mT}$. It has been shown that value of the permittivity increases linearly with volume concentration of magnetite nanoparticles. The values of permittivity decreased with increase of electric field for all concentrations which is probably caused by shifting magnetite nanoparticles to circular electrodes which means that the geometry of electric field was changed by the decrease of active part of dielectrics. The highest value of dielectric anisotropy was obtained for the highest volume concentration. This fact could mean that no aggregation of magnetite nanoparticle had appeared and good stability of magnetic fluid in electric and magnetic field was confirmed.

\section{Acknowledgments}

The authors thank to Oana Marinica, from Romanian Academy - Timisoara Division (RA-TB) for magnetic measurements of the samples. This work was supported by Slovak Academy of Sciences, in the framework of CEXNANOFLUID and by European Community, ERDF - European regional development funds 26220120003, 26220220061, 26220220064 Common project MAFINCO (Slovakia) and CNMP (Romania no. 7-018/2009) in the framework of the FP7 ERA-NET Transnational Cooperation.

\section{References}

[1] A. Espurz, J.M. Alameda, J. Phys. D, Appl. Phys. 22, 1174 (1989).

[2] A.J. Mailfert, B. Nahounou, IEEE Trans. Magn. 16, 254 (1980).

[3] O. Derriche, L. Jorat, G. Noyel, J. Monin, J. Magn. Magn. Mater. 102, 155 (1991).

[4] A. Colteu, J. Magn. Magn. Mater. 39, 28 (1983).

[5] A. Cotae, J. Magn. Magn. Mater. 39, 85 (1983).

[6] P. Kopcansky, J. Cernak, P. Macko, D. Spisak, K. Marton, J. Phys. D 22, 1410 (1989).

[7] B.Z. Kaplan, D.M. Jacobson, Nature (London) 259, 654 (1976)); doi:10.1038/259654a0.

[8] P.C. Fannin, B.K. Scaife, S.W. Charles, J. Magn. Magn. Mater. 122, 168 (1993).

[9] N.A. Yusuf, J. Shobaki, H. Abu-Safia, I. Abu-Aljarayesh, J. Magn. Magn. Mater. 149, 373 (1995).

[10] K. Marton, L. Tomčo, F. Herchl, P. Kopčanský, B. Dolník, M. Timko, M. Koneracká, in: Proc. DISEE, , ISBN 80-227-2470-X, Bratislava 2006, p. 16.

[11] D. Bica, Rom. Repts. Phys. 47, 265 (1995).

[12] S. Holotescu, F.D. Stoian, O. Marinica, L. Kubicar, P. Kopcansky, M. Timko, J. Magn. Magn. Mater. 323, 1343 (2011).

[13] J.C. Maxwell, A Treatise on Electricity and Magnetism, 2nd ed., Vol. I, Clarendon Press, Oxford 1881, p. 435.

[14] F. Herchl, K. Marton, L. Tomco, P. Kopcansky, M. Timko, M. Koneracka, I. Kolcunova, J. Phys., Condens. Matter 20, 204110 (2008).

[15] M.M. Radulescu, J. Magn. Magn. Mater. 85, 144 (1990). 